ELECTRONIC LETTER

\title{
The G6055A (G2019S) mutation in LRRK2 is frequent in both early and late onset Parkinson's disease and originates from a common ancestor
}

\author{
S Goldwurm*, A Di Fonzo*, E J Simons, C F Rohé, M Zini, M Canesi, S Tesei, A Zecchinelli, \\ A Antonini, C Mariani, N Meucci, G Sacilotto, F Sironi, G Salani, J Ferreira, H F Chien, E Fabrizio, \\ N Vanacore, A Dalla Libera, F Stocchi, C Diroma, P Lamberti, C Sampaio, G Meco, E Barbosa, \\ A M Bertoli-Avella, G J Breedveld, B A Oostra, G Pezzoli, V Bonifati
}

J Med Genet 2005;42:e (http://www.jmedgenet.com/cgi/content/full/42/11/e65). doi: 10.1136/jmg.2005.035568

Background: Mutations in the gene Leucine-Rich Repeat Kinase 2 (LRRK2) were recently identified as the cause of PARK8 linked autosomal dominant Parkinson's disease.

Objective: To study recurrent LRRK2 mutations in a large sample of patients from Italy, including early $(<50$ years) and late onset familial and sporadic Parkinson's disease. Results: Among 629 probands, 13 (2.1\%) were heterozygous carriers of the G2019S mutation. The mutation frequency was higher among familial $(5.1 \%, 9 / 177)$ than among sporadic probands $(0.9 \%, 4 / 452)(p<0.002)$, and highest among probands with one affected parent $(8.7 \%, 6)$ 69) $(p<0.001)$. There was no difference in the frequency of the G2019S mutation in probands with early $v$ late onset disease. Among 600 probands, one heterozygous R1441C but no R1441G or Y1699C mutations were detected. None of the four mutations was found in Italian controls. Haplotype analysis in families from five countries suggested that the G2019S mutation originated from a single ancient founder. The G2019S mutation was associated with the classical Parkinson's disease phenotype and a broad range of onset age (34 to 73 years).

Conclusions: G2019S is the most common genetic determinant of Parkinson's disease identified so far. It is especially frequent among cases with familial Parkinson's disease of both early and late onset, but less common among sporadic cases. These findings have important implications for diagnosis and genetic counselling in Parkinson's disease.

$P$ arkinson's disease affects more than $1 \%$ of people after the age of 65 years, and is the second most common neurodegenerative disorder after Alzheimer's disease. The disease is defined clinically by the association of bradykinesia, resting tremor, muscular rigidity, and postural instability, and pathologically by loss of dopaminergic neurones in the substantia nigra-pars compacta and other brain sites, with formation of ubiquitin containing inclusions (Lewy bodies) in the surviving neurones. ${ }^{\prime}$

The cause of the disease remains unknown in most patients, but a positive family history of Parkinson's disease is found in $\sim 15-25 \%$ of cases, and mutations in five genes have been firmly implicated in the aetiology of rare inherited forms of the disease. ${ }^{23}$

An autosomal dominant form of Parkinson's disease (PARK8) was first mapped to chromosome 12 in a Japanese family ${ }^{4}$; this linkage was later confirmed in white families. ${ }^{5}$ Recently, mutations in the gene Leucine-Rich Repeat
Kinase 2(LRRK2) (MIM *609007) were identified in PARK8 linked families. ${ }^{8}$ The LRRK2 gene encodes a predicted protein of 2527 amino acids, which has unknown function. This protein, termed dardarin, belongs to the ROCO group within the Ras/GTPase superfamily, and contains several conserved domains: an Roc (Ras in complex proteins) and a COR (C-terminal of Roc) domain, together with a leucinerich repeat, a WD40 domain, and a tyrosine kinase catalytic domain. ${ }^{9}$

To date, seven LRRK2 pathogenic mutations have been reported in autosomal dominant Parkinson's disease. Four of these mutations recurred in at least two unrelated families: Y1699C (present in two large kindreds, family " $\mathrm{A}$ " of German-Canadian ancestry, and one British kindred $)^{78}$; R1441C (found in family " $\mathrm{D}$ " of Western Nebraska origin, and another family) ${ }^{8}$; R1441G (found in several families and a few sporadic cases in the Basque population $)^{7}$; and G2019S, which we and other groups have recently identified. ${ }^{10-14}$

Mutations in the LRRK2 gene, particularly G2019S, appear to be relevant for Parkinson's disease, but the frequency of these mutations according to clinical features of the probands-such as onset age and pattern of presentation (familial or sporadic)—has not been assessed in large consecutive series of probands from homogeneous well defined populations. The frequency of known or novel LRRK2 mutations might be different in different populations; moreover, the previous studies have targeted mainly late onset Parkinson's disease series. Therefore the frequency of mutations remains unknown among early onset patients.

The penetrance of LRRK2 mutations appears strongly age related, and is probably incomplete 48101214 ; these mutations might therefore also be expected in patients with the sporadic presentation (the vast majority of cases of Parkinson's disease). It is therefore urgent to assess the prevalence and associated phenotype of the G2019S and other LRRK2 mutations in clinically and ethnically well defined series of familial and sporadic Parkinson's disease cases, including early and late onset patients.

Here, we report the first study of all four so far known recurrent $L R R K 2$ mutations in a large sample of 629 probands with Parkinson's disease ascertained at a single centre in Italy. We also analyse the haplotypes and the clinical phenotypes associated with the G2019S mutation.

Abbreviations: LD, linkage disequilibrium; SNP, single nucleotide polymorphism 
Table 1 Distribution of study sample according to 10 year onset age classes

\begin{tabular}{|c|c|c|c|c|c|c|c|}
\hline & \multicolumn{3}{|c|}{ Early onset (years) } & \multicolumn{3}{|c|}{ Late onset (years) } & \multirow[b]{2}{*}{ Total } \\
\hline & $<\mathbf{3 0}$ & $30-39$ & $40-49$ & $50-59$ & $60-69$ & $\geqslant 70$ & \\
\hline All cases & 7 & 83 & 140 & 224 & 142 & 33 & 629 \\
\hline G2019S heterozygous & - & 3 & 4 & 4 & - & 2 & 13 \\
\hline Familial & 1 & 21 & 46 & 65 & 33 & 11 & 177 \\
\hline G2019S heterozygous & - & 2 & 3 & 3 & - & 1 & 9 \\
\hline Sporadic & 6 & 62 & 94 & 159 & 109 & 22 & 452 \\
\hline G2019S heterozygous & - & 1 & 1 & 1 & - & 1 & 4 \\
\hline
\end{tabular}

\section{METHODS}

\section{Subjects and clinical analyses}

We studied 629 probands, representing two consecutive cohorts of Parkinson's disease cases with early onset disease $(<50$ years old at symptoms onset, $\mathrm{n}=230)$ or late onset disease ( $\geqslant 50$ years old at onset, $n=399$ ). The age at which the patient noticed the first symptom was considered to be the age of disease onset. Thirty three relatives affected by Parkinson's disease were also included, giving a total of 662 cases with the disease. All cases were examined and collected at the Parkinson Institute, Istituti Clinici di Perfezionamento, Milan, one of the largest referral centres for diagnosis and treatment of Parkinson's disease in Italy. Most cases were of Italian origin, but one case originated from each of the following countries: Argentina, Colombia, Ethiopia, France, Greece, Iceland, Ireland, Israel, and the United Kingdom.

The mean (SD) age at disease onset was 52.7 (10.9) years in the whole series of 629 probands, and 40.8 (5.6) years and 59.5 (6.6) years in the early onset and late onset groups, respectively. The clinical diagnosis of definite Parkinson's disease was established according to widely accepted criteria, ${ }^{15}$ and required the presence of bradykinesia and at least one of the following: resting tremor, rigidity, and postural instability; a positive response to dopaminergic therapy; and the absence of atypical features or other causes of parkinsonism.

Patients were classified as "familial" if at least one relative was reported with a formal diagnosis of Parkinson's disease among the first, second, or third degree relatives. The other probands were classified as "sporadic".

The four mutations were tested-using the same method as for the Parkinson's disease cases-in 440 Italian controls, including 304 elderly individuals free from Parkinson's disease or dementia (spouses of Parkinson's disease cases, outpatients of general practices, and blood donors, average age 66.4 (9.3) years), and 136 inpatients with cerebrovascular disease (average age 64.9 (8.4) years). The whole sample of controls (880 chromosomes) was tested for the G2019S mutation. The remaining mutations were tested in a total of 530 chromosomes. The project was approved from the local ethics authorities, and written informed consent was obtained from all subjects.

\section{Mutation analysis}

Genomic DNA was isolated from peripheral blood using standard protocols. ${ }^{16}$ The primers and polymerase chain reaction (PCR) protocol used to amplify the LRRK2 exons (Nos 31, 35, and 41) containing the C4321T (R1441C), C4321G (R1441G), the A5096G (Y1699C), and the G6055A (G2019S) mutation have been reported previously..$^{10}$ The consequences of mutations at the protein level were predicted according to the LRRK2 cDNA sequence (Genbank accession number AY792511).

About 20 ng of pooled PCR product (exons 31, 35, and 41) were purified using ExoSAP-IT (USB) and used in a primer extension reaction (SNaPshot) including the following primers: for the R1441C and the R1441G mutations in exon 31 (sense strand), 5' -agaatcacaggggaagaagaagcgc- 3 ', product size 26 base pairs (bp) (primer length plus one base); for the Y1699C mutation in exon 35 (antisense strand), 5'-taatcgattgattaatcttgaccaaaatccattggaaaa-3', product size $41 \mathrm{bp}$; for the G2019S mutation in exon 41 (antisense strand), 5' aatgctgccatcattgcaaagattgctgactac- $3^{\prime}$, product size $34 \mathrm{bp}$.

Reactions were carried out in $10 \mu \mathrm{l}$ containing $1 \mu \mathrm{l}$ SNaPshot multiplex ready reaction mix (Applied Biosystems, Foster City, California, USA); $2.5 \mu \mathrm{M}$ R144lC/ R1441G, $7.5 \mu \mathrm{M}$ Y1699C, $2.5 \mu \mathrm{M}$ G2019S extension primer, and $1 \mu \mathrm{l} 1 / 2$ term buffer (200 mM TrisHCl; $5 \mathrm{mM} \mathrm{MgCl}_{2}, \mathrm{pH}$ 9). Additional thermal cycling was undertaken for 40 cycles of 10 seconds at $95^{\circ} \mathrm{C}$, five seconds at $50^{\circ} \mathrm{C}$, and 30 seconds at $60^{\circ} \mathrm{C}$. Removal of the $5^{\prime}$-phosphoryl groups was done using 1 unit of shrimp alkaline phosphatase (SAP) (Roche Diagnostics, Monza, Italy) for 30 minutes at $37^{\circ} \mathrm{C}$.

One microlitre of SNaPshot product was diluted in $10 \mu \mathrm{l}$ Hi-Di formamide (Applied Biosystems) containing GeneScan-120 LIZ size standard (Applied Biosystems), denatured for five minutes at $95^{\circ} \mathrm{C}$, cooled on ice, and loaded on an ABI3100 Genetic Analyzer (Applied Biosystems). Fragments were analysed using GeneMapper V3.0 software (Applied Biosystems).

Negative and positive controls for the G2019S and R1441C mutations were included in all experiments. Positive controls were not available for the R1441G and the Y1699C mutation. All the mutations identified in the SNaPshot screening were confirmed by direct sequencing using a second DNA aliquot.

In one case carrying the G2019S mutation and one control, total RNA was isolated from blood cells and cDNA was prepared using standard protocols. A $251 \mathrm{bp}$ fragment of the LRRK2 CDNA spanning exons 41-42 was amplified using the following primers: forward $5^{\prime}$-cacgtagctgatggtttgagatacc-3'; reverse $5^{\prime}$-ccaaatgaataaacatcagcctgt- $3^{\prime}$.

\section{Haplotype analysis}

Nineteen intragenic and flanking markers (13 microsatellites and six single nucleotide polymorphisms (SNP)) were typed, including both known exonic and a newly discovered LRRK2 intronic SNP (IVS13+104G/A) in linkage disequilibrium (LD) with the G2019S mutation. Microsatellites were selected from the Marshfield integrated map and from Kachergus et $a l^{14}$; they were amplified by PCR using fluorescently labelled F-primers according to standard methods; fragments were loaded on an ABI3100 and analysed using the GeneMapper version 3.0 software (Applied Biosystems). Exonic and intronic LRRK2 SNPs were typed by direct sequencing using the primers and PCR conditions reported previously. ${ }^{10}$

The frequency of the IVS13+104G/A SNP was assessed in 100 chromosomes from Italian Parkinson's disease cases and 200 chromosomes of Italian controls.

We included in the haplotype analysis 12 families with the G2019S mutation detected in this series, the four families reported by us previously, ${ }^{10}$ and another two unpublished families (IT-023 and TH-08, from Italy and Morocco, 


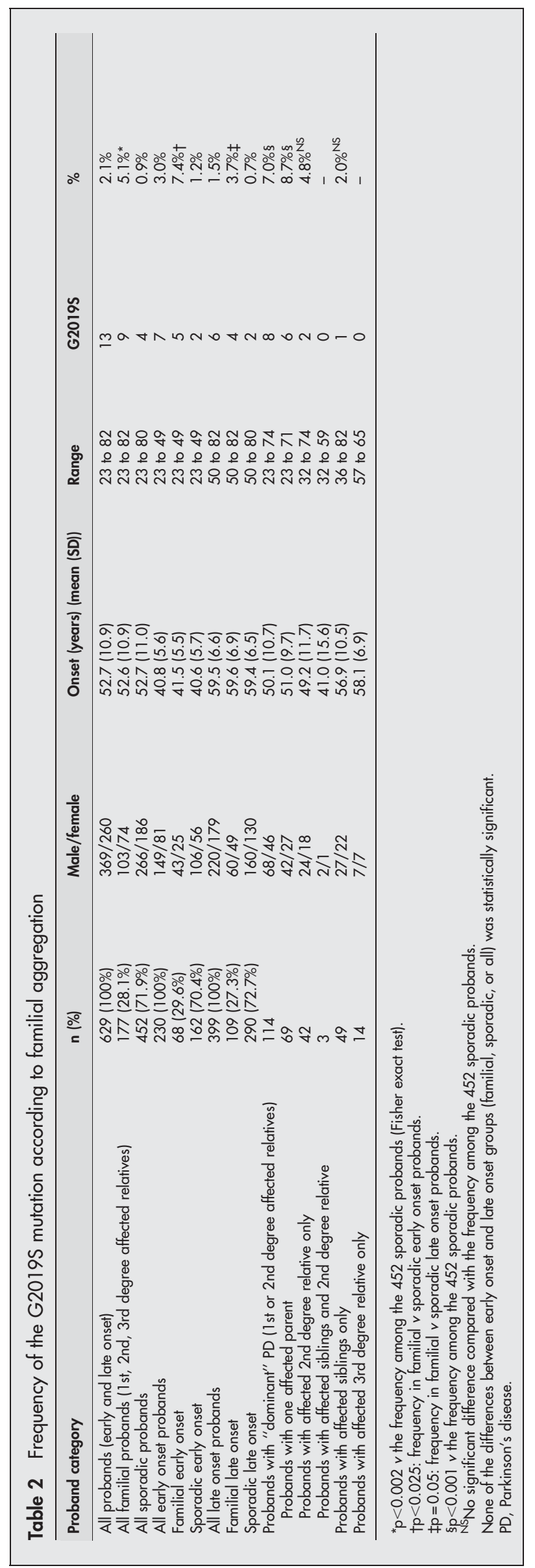


PD-1092

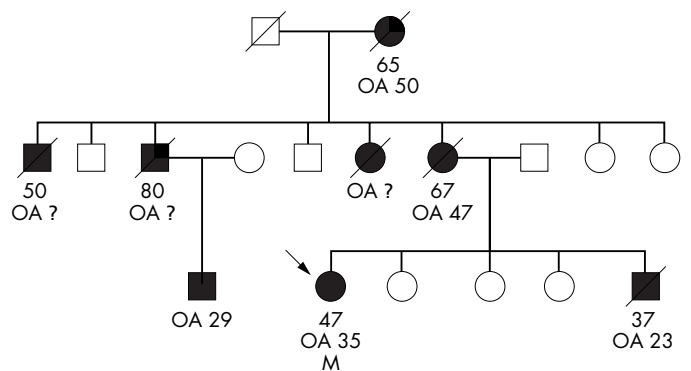

PD-1 190

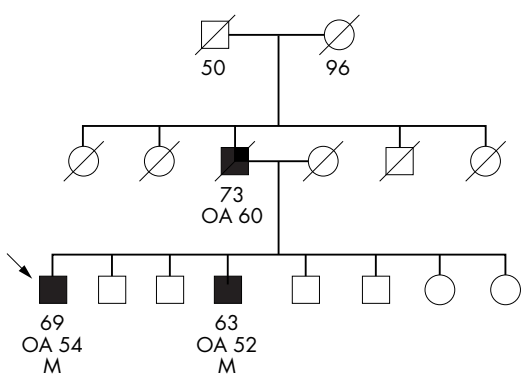

PD-499

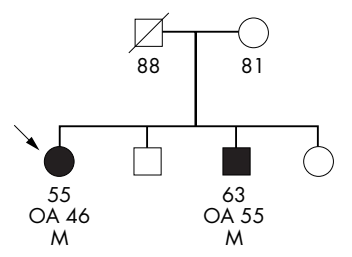

PD-07
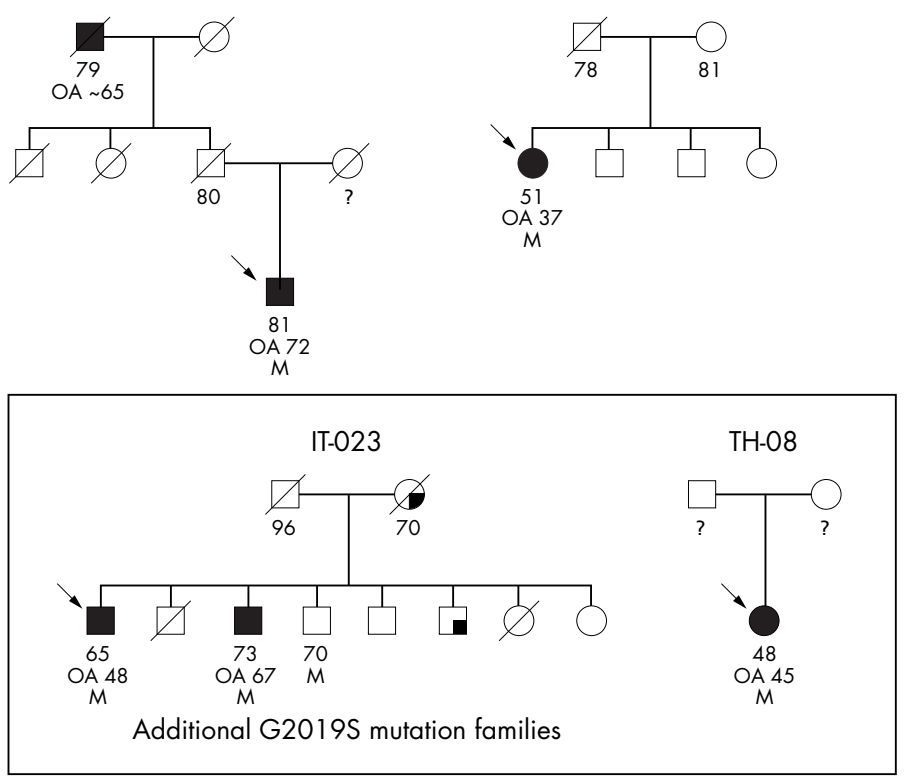

PD-869

PD-317

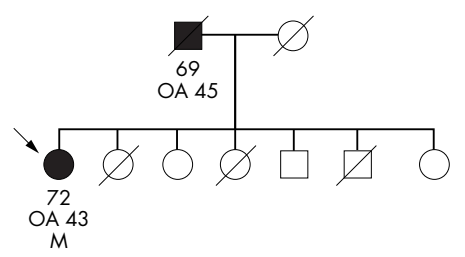

PD-516

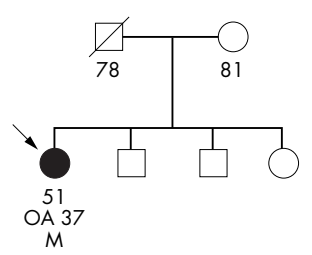

PD-794
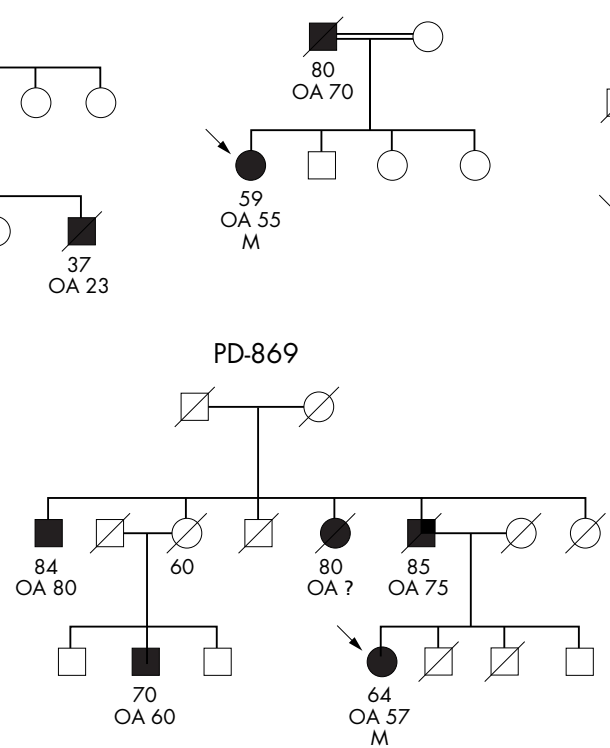

PD-902
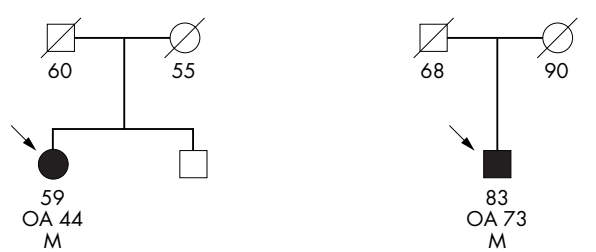

PD-768

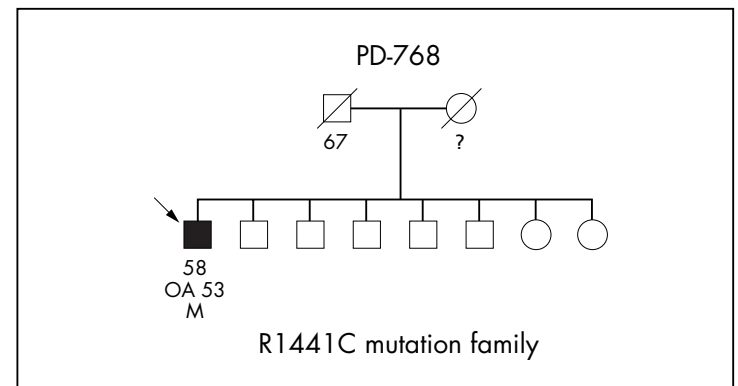

R1441C mutation family
PD-789

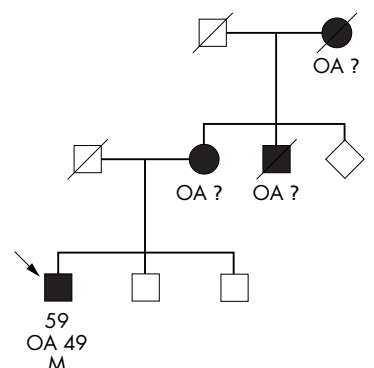

PD-1074
PD-903
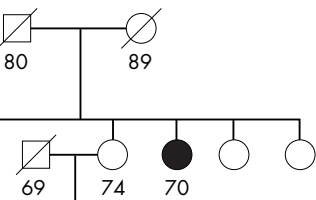

OA 56
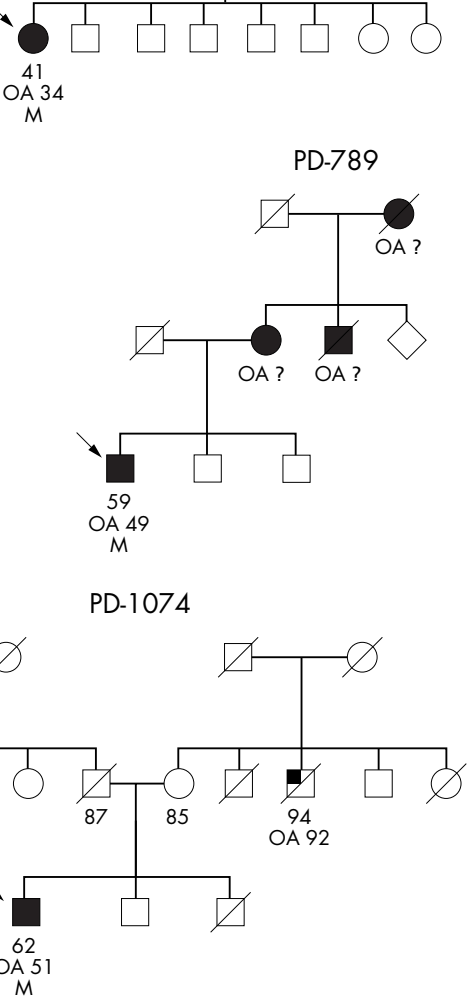

Figure 1 Simplified pedigrees of families with LRRK2 mutations. Full black symbols: individuals affected by Parkinson's disease; symbols with black upper corner: individuals affected by senile dementia; symbols with black lower corner: individuals with tremor only. To protect confidentiality the order of individuals in sibships was altered. The first number below symbols indicates age at examination or age at death (years). OA, age at disease onset (years). Question mark indicates that information is not available (individuals who lost contacts with their family). M, carrier of heterozygous $G 2019 S$ mutation. In family PD-768, M indicates the carrier of the R1441C mutation. No further individuals were known to be affected by Parkinson's disease among the more distant relatives, including the families of the sporadic Parkinson probands. Extended versions of these pedigrees are available on request. 


\section{Wild type}

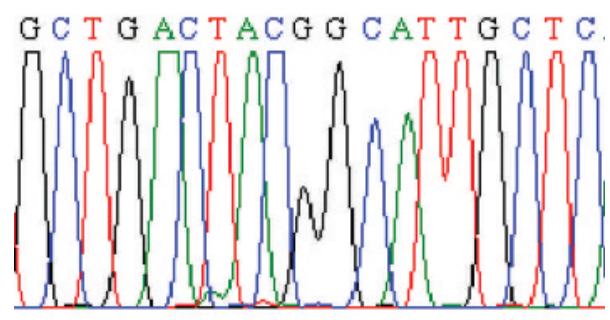

Mutant

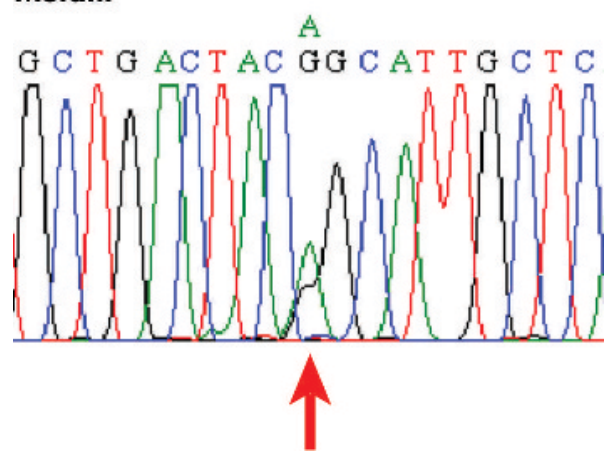

Figure 2 Electropherogram of part of $L R R K 2 \mathrm{cDNA}$ sequence from one Parkinson's disease patient and one control. The position of the heterozygous G6055A mutation (G2019S) is indicated (arrow).

respectively) identified by us from unrelated series of patients. Haplotypes were constructed manually. In four families phase could be assigned unambiguously for most markers by genotyping of trios of parents and child. In the remaining families, the phase was estimated using PHASE version 2.1. ${ }^{17}$ Haplotypes with known phase were included to improve the performance of the program. Statistical analysis was undertaken using contingency tables and the Student's $t$ test, as appropriate.

\section{RESULTS}

\section{Frequency of mutations}

The G2019S mutation was not detected in 880 control chromosomes, whereas it was identified in heterozygous state in 13 of the 629 probands (overall frequency $2.1 \%$, $\mathrm{p}<0.01 v$ controls). The distribution of probands according to onset age classes and pattern of familial aggregation is presented in tables 1 and 2 .

The carriers of the G2019S mutation included nine of 177 familial probands $(5.1 \%)$ and four of 452 sporadic probands $(0.9 \%) \quad(p<0.002$ familial $v$ sporadic). The frequency of the G2019S mutation among the familial Parkinson's disease probands remained five times higher than among the sporadic probands when early onset or late onset groups were considered separately (table 2 ).

Considering together the familial and the sporadic sample, seven of 230 early onset probands (3.0\%), and six of the 399 late onset probands $(1.5 \%)$ carried the G2019S mutation (table 2). The frequency of carriers among early onset cases remained about twofold higher than among late onset case when either the whole sample or only familial or sporadic Parkinson's disease was considered; however, the differences between early and late onset groups did not reach statistical significance.

Among 600 probands tested, there was one heterozygous for the R1441C mutation but none carrying the R1441G or the Y1699C mutation. These mutations were not observed in controls.

The simplified pedigrees are shown in fig 1 . These include the families of the 13 probands with the G2019S mutation and one with R1441C mutation identified in this study, and two unpublished families with the G2019S mutation (IT-023 and TH-08), identified from other Parkinson's disease cohorts, that were included in the haplotype study. Thirteen probands with the G2019S mutation were from Italy, one (PD-1092) was from Greece and another (TH-08) from Morocco.

In three families (PD-499, PD-1190, and IT-023), DNA was available from one affected relative; the G2019S mutation was found in heterozygous state in all these three secondary cases. The lack of DNA samples from other affected or unaffected relatives precludes further detailed analyses of cosegregation and penetrance of the mutation. The cDNA analysis from blood cells documented the expression of the mutant G2019S allele (fig 2).

\section{Haplotype analysis}

The results of the haplotype analysis are reported in fig 3. An extended shared region was present in the patients from all the families with phase assigned. For all patients with uncertain phase, the genotypes were compatible with the presence of the same haplotype (fig 3), as also predicted by the results of the PHASE program. These findings strongly suggest that the mutant G2019S allele was inherited from a common founder. The minimum size of the shared region is $\sim 160 \mathrm{~kb}$, defined by markers D12S2514 and D12S2518, while the maximum size is defined in our dataset by markers D12S2519 ( 80kb from D12S2518) and D12S2080 ( 570 kb from D12S2514).

\section{Clinical features}

Clinical features were similar in patients who carried the G2019S mutation and those who did not (table 3). Among the 15 cases detected from the consecutive cohort in this study ( 13 probands and two affected relatives) the first symptom at onset was rest tremor in five cases, bradykinesia in nine, and rigidity in one. Body distribution of signs and symptoms at onset was asymmetrical in all but one case. Bradykinesia and rigidity were present in all 15 cases on examination, while in nine cases rest tremor was documented at some time during the disease course. Decreased postural reflexes were documented in 11 cases. Response to levodopa was good in all. Motor fluctuations were observed in 13 cases, and levodopa induced dyskinesias in 12 of these. Two cases showed dystonic features. Freezing of gait was noted in 12. Severe autonomic dysfunction was not observed. Psychiatric disturbances were common: four cases had psychotic phenomena (hallucinations, delusions); two had depression years after the onset of motor symptoms, another three cases had depression at the time of onset, and in one case depression occurred seven years before the onset of motor symptoms.

Dementia was present in only one case. Sleep disturbances were also common, present in nine cases. In one case, amelioration of symptoms after sleep was noted (sleep benefit). Three cases were treated with deep brain stimulation, and one with thalamolysis.

In the patient carrying the R1441C mutation, Parkinson's disease started with asymmetrical rest tremor, later followed by bradykinesia, rigidity, and postural instability. Freeezing of gait, levodopa induced motor fluctuations, and dyskinesias also developed. Depression occurred three years before the onset of motor symptoms. 

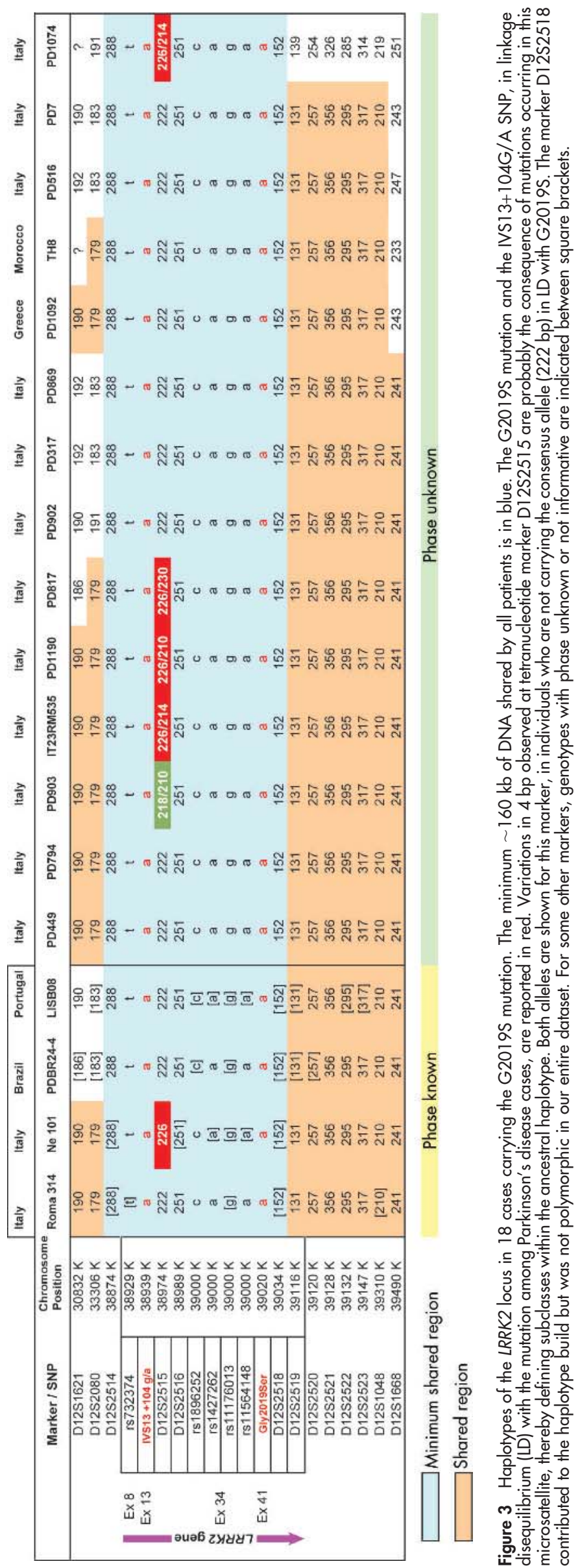

\section{DISCUSSION \\ Frequency of LRRK2 mutations in Parkinson's disease}

This is the first comprehensive study of LRRK2 recurrent mutations targeting large groups of Italian cases with early onset and late onset Parkinson's disease, with familial as well as sporadic presentation. We found a frequent occurrence of the G2019S mutation. On the other hand, the R1441C, R1441G, and Y1699C mutation were rare, suggesting they are not a relevant cause of Parkinson's disease in the Italian population. In addition to Italy, Portugal, and Brazil, and the countries reported by others, ${ }^{10-14}$ we expand the presence of the G2019S mutation to Parkinson's disease cases from Greece and Morocco.

In the initial studies, the G2019S mutation was found in $\sim 3-6 \%$ of selected samples with familial Parkinson's disease (autosomal dominant families, and sibling pairs) from several European and North American countries, and in $\sim 1 \%$ of sporadic Parkinson's disease cases from the United Kingdom, while it was absent in more than 4000 control

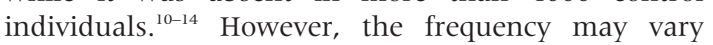
considerably between populations-recent studies suggest a very high prevalence in North African and a very low prevalence in Asian populations. ${ }^{18} 19$

The pathogenic role of the G2019S mutation is further supported by the observation that the G2019 residue is extremely conserved in human kinase domains and in all dardarin homologues. ${ }^{20}$

Here we report the frequency of G2019S in a large sample of clinically and ethnically well defined patients, showing that G2019S is significantly more frequent among the cases with familial Parkinson's disease than among those with sporadic disease, further supporting the pathogenic role of this mutation in Parkinson's disease inheritance. The phenotype associated with the mutation encompasses early and late onset Parkinson's disease, and we show here for the first time that this mutation is also common among cases with onset before the age of 50 years. However, as late onset disease represents the vast majority of cases, it is anticipated that a larger number of patients with this mutation will be identified among the cases with late onset classical Parkinson's disease.

\section{Origin of the G2019S mutation from a common founder}

Our haplotype analysis strongly suggests that the G2019S mutation is transmitted from a single ancient founder. This confirms the results of a previous study, ${ }^{14}$ and refines the size of the shared region on the $3^{\prime}$ end of the LRRK2 gene, excluding markers D12S2519 and D12S2520. More importantly, in our data the $\sim 160 \mathrm{~kb}$ minimum shared region spans the promoter and most of the LRRK2 gene, suggesting that variation at the promoter or other cis-acting regulatory elements are not important determinants of the phenotypic variation observed among G2019S carriers. However, variants at regulatory elements in the other allele might play a modifier role. In the previous study the minimum shared region was reduced to $145 \mathrm{~kb}$ from marker D12S2515 to D12S2521, thereby excluding the promoter and the first 21 exons and 20 introns of LRRK2. ${ }^{14}$ However, our data suggest that D12S2515 is a highly unstable microsatellite, and the observed data in this study and the previous study ${ }^{14}$ are also compatible with mutations occurring in this 
Table 3 Clinical features in carriers and non-carriers of the G2019S mutation

\begin{tabular}{llrll}
\hline & Carriers & $\mathbf{n}$ & Non-carriers & $\mathbf{n}$ \\
\hline Onset age (years) & $50.5(11.6)$ & 15 & $52.7(10.9)$ & 615 \\
Onset age, women (years) & $43.9(8.7)^{*}$ & 8 & $53.9(10.7)$ & 254 \\
Onset age, men (years) & $58.0(10.1)$ & 7 & $51.9(11.0)$ & 361 \\
Disease duration† (years) & $11.4(5.8)$ & 15 & $10.4(6.3)$ & 615 \\
Disease duration, women (years) & $12.1(7.8)$ & 8 & $10.3(5.9)$ & 254 \\
Disease duration, men (years) & $10.6(2.2)$ & 7 & $10.5(6.6)$ & 361 \\
\hline Values are mean (SD). & & & \\
$+Y e a r s$ from the age at onset of symptoms to the age at last examination. & & \\
* $\mathrm{P}<0.02 v$ G2019S het. male carriers (Student's $t$ test). & & \\
\end{tabular}

polymorphic marker instead of recombination events. We propose that alleles at D12S2515 define a cluster of subhaplotypes in the context of the ancestral G2019S bearing haplotype. The presence of the newly discovered IVS13+104A variant in all carriers of the mutant haplotype supports the contention that the shared region extends beyond the D12S2515 marker. We did not observe the IVS13+104A variant in any Italian Parkinson's disease cases, which do not carry the G2019S mutation (50 cases tested), and we have observed it in only three of 100 Italian controls (allele frequency $\sim 1.5 \%$ ) (the three controls were also sequenced and confirmed to be non-carriers of G2019S). The low frequency of the haplotype carrying the IVS13+104A variant in the general population also strongly suggests that G2019S originated from a single ancestor. The evidence of a common founder for this mutation in cases from many populations suggests that the mutant allele is very ancient.

\section{The clinical phenotype associated with G2019S}

The phenotype associated with the G2019S mutation in this and other studies is broad, encompassing a wide range of onset ages (from 34 to 73 years in this study), and a wide spectrum of penetrance, resulting in pattern ranging from sporadic presentation to autosomal dominant, highly penetrant familial aggregation. Pedigree inspection in our sporadic mutant probands (five carrying G2019S and one carrying R1441C) reveals that four of the 12 parents died before the age of 73 years, the latest onset age known in our patients with these mutations, including both parents of proband PD-817; information was unavailable for three parents, including both parents of proband TH-08. For the remaining five parents (both parents for probands PD-1074 and PD-516) the age at death or at examination was later than 73, and these might represent examples of nonpenetrance of the G2019S mutation. For two more probands (PD-07 and PD-903) with unaffected parents but affected second degree relatives, the "transmitting" parent also died or is still alive at an age greater than 73 . These observations strongly suggest that the penetrance and phenotype associated with this mutation might be markedly modified by other genetic or non-genetic factors. Future studies must address this issue, which complicates the genetic counselling of Parkinson's disease patients with LRRK2 mutations.

In this study, the average disease onset and duration showed no differences between the patients who carried the G2019S mutation and those who did not (table 3). However, female patients carrying the mutation $(n=8)$ had an age of onset that was almost 10 years earlier than male patients with the mutation $(\mathrm{n}=7)(\mathrm{p}<0.02$, Student's $t$ test $)$ (table 3$)$; if the other carriers of the same mutation detected in our previous study, ${ }^{10}$ with accurate onset age data available, are considered together, the difference remains significant (women 47.1 (10.3) years, $\mathrm{n}=17$; men 56.5 (10.5) years, $\mathrm{n}=11 ; \mathrm{p}<0.03)$. Larger numbers of cases are needed to substantiate this observation; however, it is possible that the penetrance of the G2019S mutation is higher or the onset earlier in female carriers. Further studies are also needed to assess prospectively the rate of progression of the disease associated with this and other LRRK2 mutations.

Dementia is within the phenotypical spectrum of LRRK2 mutations. ${ }^{821}$ The fact that dementia is rare in carriers of the G2019S mutation in this and previous studies suggests that the phenotype associated with this mutation is that of classical Parkinson's disease. However, our study targeted patients with the pure Parkinson's disease phenotype; the presence of the G2019S and other LRRK2 mutations should be investigated among patients with Parkinson's diseasedementia, or dementia with Lewy bodies.

\section{Conclusions}

Our study delineates the G2019S mutation in LRRK2 as the most important single genetic determinant of Parkinson's disease so far identified and provides sound evidence that this mutation originated from a common founder. G2019S is especially frequent among cases with familial Parkinson's disease of both early and late onset, but it also occurs-albeit more rarely-among patients with sporadic Parkinson's disease. Understanding the mechanisms of the disease caused by G2019S and other LRRK2 mutations might provide important clues for the dissection of the Parkinson's disease pathogenesis and for designing novel therapeutic strategies. The identification of a first, frequent genetic determinant of Parkinson's disease also has important implications for the diagnosis and genetic counselling of this disease.

\section{ACKNOWLEDGEMENTS}

We thank all the patients and family relatives for their contribution, Dr Francesca Sciacca, National Neurological Institute "C Besta", Milan, Italy, for providing some of the control samples, and Tom de Vries-Lentsch, Erasmus MC Rotterdam, for artwork. The DNA samples contributed by the Parkinson Institute - Istituti Clinici di Perfezionamento, Milan, Italy, were from the "Human genetic bank of patients affected by Parkinson disease and parkinsonisms", supported by Telethon grant No GTF03009. The study was supported by grants from the Internationaal Parkinson Fonds (Netherlands) and the National Parkinson Foundation (USA) to VB.

\section{Authors' affiliations}

S Goldwurm, M Zini, M Canesi, S Tesei, A Zecchinelli, A Antonini, C Mariani, N Meucci, G Sacilotto, G Pezzoli, Parkinson Institute, Istituti Clinici di Perfezionamento, Milan, Italy

A Di Fonzo, Centro Dino Ferrari, Department of Neurological Sciences, University of Milan, and Foundation "Ospedale Maggiore Policlinico, Mangiagalli e Regina Elena", Milan

F Sironi, Molecular Genetics Laboratory, IRCCS Ospedale Maggiore Policlinico, Mangiagalli e Regina Elena, Milan

G Salani, Neuroimmunology Unit, San Raffaele Scientific Institute, Milan E J Simons, C F Rohé, A M Bertoli-Avella, G J Breedveld, B A Oostra, V Bonifati, Department of Clinical Genetics, Erasmus MC, Rotterdam, Netherlands

J Ferreira, C Sampaio, Neurological Clinical Research Unit, Institute of Molecular Medicine, Lisbon, Portugal

H F Chien, E Barbosa, Department of Neurology, University of São Paulo, São Paulo, Brazil 
E Fabrizio, G Meco, Department of Neurological Sciences, La Sapienza University, Rome, Italy

N Vanacore, National Centre of Epidemiology, National Institute for Health, Rome

A Dalla Libera, Neurology Division, "Boldrini" Hospital, Thiene, Italy F Stocchi, IRCSS Neuromed, Pozzilli, Italy

C Diroma, P Lamberti, Department of Neurology, University of Bari, Italy

Competing interests: none declared

*These authors contributed equally to the work.

Correspondence to: Dr V Bonifati, Department of Clinical Genetics, Erasmus MC Rotterdam, PO Box 1738, 3000 DR Rotterdam, Netherlands; v.bonifati@erasmusmc.nl

Received 3 June 2005

Revised version received 22 June 2005

Accepted for publication 27 June 2005

\section{REFERENCES}

1 Lang $A E$, Lozano AM. Parkinson's disease. First of two parts. N Engl J Med 1998;339:1044-53.

2 Bonifati V, Oostra BA, Heutink P. Unraveling the pathogenesis of Parkinson's disease - the contribution of monogenic forms. Cell Mol Life Sci 2004;61:1729-50.

3 Dawson TM, Dawson VL. Molecular pathways of neurodegeneration in Parkinson's disease. Science 2003;302:819-22.

4 Funayama M, Hasegawa K, Kowa H, Saito M, Tsuji S, Obata F. A new locus for Parkinson's disease (PARK8) maps to chromosome 12p11.2-q13.1. Ann Neurol 2002;51:296-301.

5 Zimprich A, Muller-Myhsok B, Farrer M, Leitner P, Sharma M, Hulihan M, Lockhart P, Strongosky A, Kachergus J, Calne DB, Stoessl J, Uitti RJ, Pfeiffer RF, Trenkwalder C, Homann N, OHt E, Wenzel K, Asmus F, Hardy J, Wszolek Z, Gasser T. The PARK8 locus in autosomal dominant parkinsonism: confirmation of linkage and further delineation of the disease-containing interval. Am J Hum Genet 2004;74:11-19.

6 Paisan-Ruiz C, Saenz A, de Munain AL, Marti I, Martinez Gil A, MartiMasso JF, Perez-Tur J. Familial Parkinson's disease: clinical and genetic analysis of four Basque families. Ann Neurol 2005;57:365-72.

7 Paisan-Ruiz C, Jain S, Evans EW, Gilks WP, Simon J, van der Brug M, de Munain AL, Aparicio S, Gil AM, Khan N, Johnson J, Martinez JR, Nicholl D, Carrera IM, Pena AS, de Silva R, Lees A, Marti-Masso JF, Perez-Tur J, Wood NW, Singleton AB. Cloning of the Gene Containing Mutations that Cause PARK8-Linked Parkinson's Disease. Neuron 2004;44:595-600.

8 Zimprich A, Biskup S, Leitner P, Lichtner P, Farrer M, Lincoln S, Kachergus J, Hulihan M, Uitti RJ, Calne DB, Stoessl AJ, Pfeiffer RF, Patenge N, Carbajal IC,
Vieregge $P$, Asmus F, Muller-Myhsok B, Dickson DW, Meitinger T, Strom TM, Wszolek ZK, Gasser T. Mutations in LRRK2 cause autosomal-dominant parkinsonism with pleomorphic pathology. Neuron 2004;44:601-7.

9 Bosgraaf L, Van Haastert PJ. Roc, a Ras/GTPase domain in complex proteins. Biochim Biophys Acta 2003;1643:5-10.

10 Di Fonzo A, Rohe CF, Ferreira J, Chien HF, Vacca L, Stocchi F, Guedes L, Fabrizio E, Manfredi M, Vanacore N, Goldwurm S, Breedveld G, Sampaio C, Meco G, Barbosa E, Oostra BA, Bonifati V. A frequent LRRK2 gene mutation associated with autosomal dominant Parkinson's disease. Lancet 2005;365:412-15.

11 Hernandez DG, Paisan-Ruiz C, Mclnerney-Leo A, Jain S, MeyerLindenberg A, Evans EW, Berman KF, Johnson J, Auburger G, Schaffer AA, Lopez GJ, Nussbaum RL, Singleton AB. Clinical and positron emission tomography of Parkinson's disease caused by LRRK2. Ann Neurol 2005;57:453-6.

12 Nichols WC, Pankratz N, Hernandez D, Paisan-Ruiz C, Jain S, Halter CA Michaels VE, Reed T, Rudolph A, Shults CW, Singleton A, Foroud T. Genetic screening for a single common LRRK2 mutation in familial Parkinson's disease. Lancet 2005;365:410-12.

13 Gilks WP, Abou-Sleiman PM, Gandhi S, Jain S, Singleton A, Lees AJ, Shaw K, Bhatia KP, Bonifati V, Quinn NP, Lynch J, Healy DG, Holton JL, Revesz T, Wood NW. A common LRRK2 mutation in idiopathic Parkinson's disease. Lancet 2005;365:415-16

14 Kachergus J, Mata IF, Hulihan M, Taylor JP, Lincoln S, Aasly J, Gibson JM, Ross OA, Lynch T, Wiley J, Payami H, Nutt J, Maraganore DM, Czyzewski K, Styczynska M, Wszolek ZK, Farrer MJ, Toft M. Identification of a novel LRRK2 mutation linked to autosomal dominant parkinsonism: evidence of a common founder across European populations. Am J Hum Genet 2005;76:672-80.

15 Hughes AJ, Daniel SE, Kilford L, Lees AJ. Accuracy of clinical diagnosis of idiopathic Parkinson's disease: a clinico-pathological study of 100 cases. J Neurol Neurosurg Psychiatry 1992;55:181-4.

16 Miller SA, Dykes DD, Polesky HF. A simple salting out procedure for extracting DNA from human nucleated cells. Nucleic Acids Res 1988;16:1215.

17 Stephens M, Smith NJ, Donnelly P. A new statistical method for haplotype reconstruction from population data. Am J Hum Genet 2001;68:978-89.

18 Tan EK, Shen H, Tan LC, Farrer M, Yew K, Chua E, Jamora RD, Puvan K, Puong KY, Zhao Y, Pavanni R, Wong MC, Yih Y, Skipper L, Liu JJ. The G2019S LRRK2 mutation is uncommon in an Asian cohort of Parkinson's disease patients. Neurosci Lett 2005 [Epub ahead of print] Jun 12, DOI:10.1016/j.neulet.2005.04.103

19 Lesage S, Ibanez P, Lohmann E, Agid Y, Durr A, Brice A. The G2019S LRRK2 Mutation in autosomal dominant European and North African Parkinson's disease is frequent and its penetrance is age-dependent [abstract]. Neurology 2005;64:1826.

20 Manning G, Whyte DB, Martinez R, Hunter T, Sudarsanam S. The protein kinase complement of the human genome. Science 2002;298:1912-34.

21 Wszolek ZK, Cordes M, Calne DB, Munter MD, Cordes I, Pfeifer RF. [Hereditary Parkinson disease: report of three families with dominant autosomal inheritance]. Nervenarzt 1993;64:331-5. 